\title{
14 Kritik und Verbesserungsmöglichkeiten
}

Die obige Darstellung der Rechtslage zeigt, dass die Vorgaben der DSGVO einen sinnvollen Ausgleich zwischen der Forschungsfreiheit und dem Datenschutz ermöglichen. Zugleich ist aber erkennbar, dass die wegen der Öffnungsklauseln zulässigen nationalen Regelungen die europäischen Vorgaben nur unzureichend umsetzen. Im Folgenden sollen die sich daraus ergebenden Probleme bei der Umsetzung von Datenschutz in der medizinischen Forschung dargelegt werden. Daraus werden dann Vorschläge abgeleitet, mit denen insbesondere gesetzgeberisch bestehende Defizite behoben werden können.

\subsection{Defizite}

Von vielen Seiten wird immer wieder beklagt, dass Datenschutzregelungen die medizinische Forschung behindern würden. Dabei werden die bestehenden Regelungen zumeist mit dem grundrechtlichen Anliegen des Datenschutzes in einen Topf geworfen. ${ }^{910}$

Der Datenschutz im Gesundheitsbereich generell wie auch der Datenschutz im Bereich der Medizinforschung leiden darunter, dass sie einerseits über-, andererseits unterreguliert sind: Die Überregulierung ergibt sich daraus, dass Regelungen auf vielen Ebenen und aus verschiedenen Regelwerken anzuwenden sind: auf EUEbene - DSGVO, auf nationaler Ebene - BDSG, das BCB ( $\$ \$$ 630a ff.), $\$ 203$ StCB, die

910 Nachweise, auch für die Undifferenziertheit der Kritik, bei Thüsing/Rombey NZS 2019, $201 f$. 
Sozialgesetzbücher, viele bereichsspezifische Regelungen, auf Landesebene - die LDSG, Landeskrankenhausgesetze und viele weitere spezialgesetzliche Regelungen sowie die Berufsordnungen der Landesärztekammern, die sich weitgehend an der MBOÄ orientieren. ${ }^{911}$ Die Regelungen sind nur begrenzt aufeinander abgestimmt, auch soweit deren Anpassung an die DSGVO erfolgte. Es wurde vorrangig eine formelle und keine inhaltliche Anpassung vorgenommen. Die Regelungen führen zu einem normativen Flickenteppich. Sie sind unübersichtlich, in sich teilweise widersprüchlich und für Anwender in der Praxis oft nicht oder nur unter großem Aufwand umzusetzen. ${ }^{912}$

Die Unterregulierung insbesondere im Forschungsbereich ergibt sich daraus, dass eine Vielzahl von Fragen ungeklärt bleibt, so etwa der Bereich der Genforschung, der Umgang mit Biobanken, aber auch die Konkretisierung der teilweise sehr allgemeinen strafrechtlichen und datenschutzrechtlichen Vorgaben, etwa die Feststellung eines privilegierten Forschungsvorhabens. Soweit Gesetze eine Abwägung zwischen den Betroffeneninteressen und den Forschungsinteressen vorsehen, werden keine handhabbaren Abwägungskriterien vorgegeben. ${ }^{913}$ Hinzu kommen große Vollzugsdefizite insbesondere im Bereich der Datenschutzaufsicht. ${ }^{914}$

Den geltenden Regelungen zur (medizinischen) Forschung ist gemein, dass eine Datennutzung ohne Einwilligung der Betroffenen nur im Ausnahmefall auf der Crundlage einer Güterabwägung erlaubt ist. Die Einwilligung hat absoluten Vorrang als rechtliche Legitimation. Dieser Grundsatz folgt dem Wunsch, dass der Betroffene idealerweise selbst bestimmen soll, wer worüber mit seinen Daten forschen darf. Dieses Kernprinzip der informationellen Selbstbestimmung ist unbestritten. Allerdings kann es nicht in allen Lebensbereichen uneingeschränkt realisiert werden, weil in Zivilgesellschaften stets eine Balance zwischen individuellen und gemeinschaftlichen Belangen herzustellen ist. Im überwiegenden Allgemeininteresse müssen Abweichungen zulässig sein, wobei es allerdings (gesetzlicher) Regeln bedarf, die die Wahrung der Verhältnismäßigkeit garantieren. ${ }^{915}$ Von diesem Ansatz ist die DSGVO geprägt. Insofern sind im Zuge einer Neuregulierung der Nutzung medizinischer Daten für Forschungszwecke organisatorische und verfahrensrechtliche Vorkehrungen zu treffen, die einer Verletzung von Persönlichkeitsrechten effektiv vorbeugen.

Eine wirksame Einwilligung bzw. Schweigepflichtentbindung setzt voraus, dass sie informiert erfolgt, d.h. auf hinreichend präzisen Informationen darüber basiert, welche Stelle für welche Zwecke mit welchen Daten forschen können soll. Aus den unter Kap. 7.2 genannten Gründen fehlt eine klare Information aber oftmals.

911 Kritisch hierzu schon Kilian NJW 1998, 788.

912 Datenethikkommission, 20 (These 17); Sachverständigenrat, S. XXVIII (These 23), 200, 205; Weichert 2018, Kap. 10.8; Weichert MedR 2019, 624; Weichert/Krawczak MIBE 2019, Vol. 15(1), 3f./8; Kühling, 33; BKL-R, 218; ähnlich BT-Drs. 19/18111, 27; siehe auch den systematischen Überblick bei Weichert in Dockweiler/Fischer, ePublicHealth, 2019, 31, 42; Weichert in Bär/Grädler/Mayr, Digitalisierung im Spannungsfeld von Politik, Wirtschaft, Wissenschaft und Recht, Bd. 1, 2018, 558f.; Dierks 2019, 11; ders. 2020, 4f.; Graf von Kielmansegg in TMF, 90; Hense in Sydow, Bundesdatenschutzgesetz, 2020, \$ 27 Rn. 5.

913 Graf von Kielmansegg in TMF, $106 \mathrm{ff}$.

914 Datenethikkommission, 20 (These 18); Weichert MedR 2019, 624; zu den Defiziten bei der Strafverfolgung Weichert in AG Medizinrecht in DAV/IMR, Aktuelle Entwicklungen im Medizinstrafrecht, 2018, 119f.

915 Datenethikkommission, 96; Thüsing/Rombey NZS 2019, 203; Krawczak/Semler/Zenke/Strech/Graf von Kielmansegg in TMF, $129 f$. 
Häufig lässt sich das wissenschaftliche Potenzial von Gesundheitsdaten nur durch eine einrichtungsübergreifende (möglicherweise weltweite) Zusammenführung der Daten angemessen ausschöpfen, etwa bei der Erforschung seltener Erkrankungen. Unter Umständen bedarf es darüber hinausgehend sogar einer interdisziplinären stellenübergreifenden wissenschaftlichen Zusammenarbeit. ${ }^{916}$ Die Zusammenführung von Daten ist über Forschungsnetzwerke, Krankheitsregister oder andere Großprojekte realisierbar. ${ }^{917}$ Allerdings gibt es hierfür, abgesehen von den Spezialfällen der Krebsregistergesetze, dem Implantateregister (\$ 1 Abs. 2 Nr. 6 IRegG) und dem Transplantationsregister ( $\$ 15 \mathrm{a}$ TPG), keine expliziten gesetzlichen Grundlagen. Die Rechtmäßigkeit der Datennutzung gründet vielmehr allein auf der Einwilligung der Betroffenen mit dem Vorbehalt, dass Art und Umfang der Datenzusammenführung zum Zeitpunkt der Einwilligung meist völlig unbekannt sind.

Für die betreffenden Datenquellen gibt es in der Regel bereichsspezifische Vorgaben, die die Datennutzung bzw. -weitergabe an eine Genehmigung oder zumindest Kenntnisnahme durch Ministerien oder Datenschutzbehörden knüpfen. ${ }^{918}$ Die daraus resultierenden administrativen Anforderungen bedeuten einen hohen Aufwand für die Forscher und führen wegen rechtlicher Unwägbarkeiten leicht zu Verunsicherungen. ${ }^{919}$ Bisweilen können sich einschlägige Regelungen oder deren Auslegung durch Aufsichtsbehörden auf unterschiedlichen Ebenen widersprechen (z.B. bei Forschungsprojekten, für die zugleich Bundes- und Landesgesetze anwendbar sind), ${ }^{920}$ was Forscher dazu bringt, unabsichtlich und oft unwissentlich gegen rechtliche Vorgaben zu verstoßen. ${ }^{921}$

Über die Erfüllung der datenschutzrechtlichen Vorgaben hinaus müssen bei vielen medizinischen Forschungsvorhaben entsprechend $\mathbb{1} 15$ MBOÄ auch Ethikkommissionen einbezogen werden. Dauer und Ergebnis der damit verbundenen Beratungsund Genehmigungsprozesse sind oftmals schwer einschätzbar. Außerdem kommt es in vielen Belangen zur Doppelung von Aufgaben und Infrastrukturen, da ethische und datenschutzrechtliche Erwägungen teilweise identische Schutzziele verfolgen (Würdeschutz, Persönlichkeitsschutz, sonstiger Grundrechtsschutz). Beide Verfahren fordern letztlich eine Abwägung von Forschungsinteressen und Betroffeneninteressen; sie unterscheiden sich insbesondere in der Zusammensetzung des „Spruchkörpers" und der dort präsenten Expertise. ${ }^{922}$

Das neue BDSG und die neuen Landesdatenschutzgesetze schaffen weder Rechtssicherheit noch Rechtsklarheit, da der Flickenteppich von Regelungen zur Forschungsdatenverarbeitung mitsamt dem bereichsspezifischen Recht fortbesteht. ${ }^{923}$ Die Zweigleisigkeit des Datenschutzes zwischen SCB und sonstigem Recht verstärkt die Verunsicherung und legt zugleich eine Anpassung nahe. ${ }^{924}$ Gemäß Art. 9 Abs. 3

916 Datenethikkommission, 75; Deutscher Ethikrat, 11ff. (Thesen 15-17, 19, 74), 60ff.; GMDS, 5.

917 GMDS, 10.

918 Z.B. $\$ 75$ Abs. 4 SGB X, \ 27a Abs. 2 ThürKHG.

919 Dierks 2019, 62ff.

920 So die Begründung zu $\$ 287$ a SGB V des „Gesetzes zum Schutz der Bevölkerung bei einer epidemischen Lage von nationaler Tragweite" v. 24.03.2020, BT-Drs. 19/18111, 27; Graf von Kielmansegg in TMF, 116.

921 Dierks 2019, $86 f$.

922 Graf von Kielmansegg in TMF, $117 f$.

923 Datenethikkommission, 125; Graf von Kielmansegg in TMF, 104.

924 Zum Novellierungsdarf im SGB Thüsing/Rombey NZS 2019, 205. 
DSGVO bleibt das nationale Recht zu Berufsgeheimnissen erhalten, was getreu dem in Deutschland bestehenden Zwei-Schranken-Prinzip bedeutet, dass die Weiterverarbeitung von Berufsgeheimnissen (wie etwa des Patientengeheimnisses) durch Dritte neben der datenschutzrechtlichen Erlaubnis eine zusätzliche Offenbarungsbefugnis verlangt. Die völlig offen formulierten Regelungen, etwa in $\$ 27$ BDSG, können diesbezüglich nicht zufriedenstellen.

Die fehlende Rechtssicherheit betrifft auch die Frage, welche Forschungsprojekte überhaupt in den Genuss der in der DSGVO und in nationalstaatlichen Regelungen vorgesehenen Privilegierungen kommen können. Es fehlt an einer klaren materiellen Definition privilegierter Forschung. Zudem fehlt es an einem Verfahren, durch das die Voraussetzungen für die Privilegierung verbindlich festgestellt werden können. Selbst der jüngste Vorstoß der Bundespolitik, die medizinische Forschung durch neue Regelungen zum Transparenzregister ( $\$ \mathbb{S}$ 303a SCB V) zu stärken, hat dieses Problem nicht aufgegriffen. ${ }^{925}$

Die bestehende Rechtsunsicherheit könnte auf der Grundlage der DSGVO beseitigt werden. Kritisch zu bewerten sind dagegen Vorschläge des Deutschen Ethikrates, die unter dem Stichwort der „Datensouveränität“ eine Abkehr von den Grundsätzen der Zweckbindung und der Datenminimierung propagieren. ${ }^{926}$ Mit der DSGVO wurde ein supranationaler Rechtsrahmen geschaffen, in dem Gesundheitsdaten (Art. 4 Nr. 15 DSGVO) weiterhin einen hohen Schutz genießen (Art. 9 DSGVO). Die DSGVO anerkennt aber auch das hohe öffentliche Interesse an der Weiterverarbeitung zu Forschungszwecken und erklärt deshalb diese nicht länger als unvereinbar mit dem ursprünglichen Erhebungszweck (Art. 5 Abs. 1 lit. b DSGVO). Eine wissenschaftliche Verarbeitung personenbezogener Gesundheitsdaten ist zulässig, wenn „angemessene und spezifische Maßnahmen zur Wahrung der Grundrechte und Interessen der betroffenen Person" bestehen (Art. 9 Abs. 2 lit. j, 89 DSGVO). Leider wurde die Hoffnung auf eine Vereinheitlichung und Modernisierung der Datenschutzregelungen in Deutschland zur medizinischen Forschung dadurch getrübt, dass die Gesetzgeber die bisher gültigen Regelungen nur unwesentlich geändert haben. ${ }^{927}$

\subsection{Nationaler Regelungsvorschlag}

Diese Defizite veranlassten den Autor und den Medizininformatiker Michael Krawzcak, zunächst in einem engen Kreis von Kollegen aus den Bereichen Medizinforschung und Datenschutz eine Diskussion über den rechtlichen Reformbedarf zu führen. Die Ergebnisse wurden in ein Handlungskonzept überführt und bundesweit an 50 einschlägige Adressaten aus einer ausgewählten Fachöffentlichkeit von Medizinforschern (u.a. Rat für Informationsinfrastruktur, Wissenschaftsrat, Deutsches Netzwerk Versorgungsforschung), Medizinfachverbänden (u.a. Bundesärztekammer), Datenschützern (u.a. Konferenz der Datenschutzbeauftragten des Bundes und der Länder, Gesellschaft für Datenschutz und Datensicherheit), Verbraucherschützern (u.a. Verbraucherzentrale Bundesverband), Verwaltung (zuständige Bundesministe-

925 Digitale-Versorgung-Gesetz v. 09.12.2019, BGBI. S. 2562; Riechert DANA 2019, 211f.; Weichert DANA 2020, 25; ders. MedR 2020, 539ff.; Schulz SGb 2020, 536ff.; Bretthauer/Spiecker, IZ 2020, $990 f f$.

926 Deutscher Ethikrat, u.a. 22f.; kritisch und richtig dazu Kühling DuD 2020, $182 f f$.

927 Weichert/Krawczak MIBE 2019, Vol. 15(1), 3/8. 
rien) und Politikern (Bundestagsfraktionen) zur Stellungnahme übermittelt. Auf Grundlage der Rückmeldungen wurde das Konzept modifiziert und anschließend zur allgemeinen politischen Diskussion gestellt. ${ }^{928}$ Das überarbeitete Konzept war u.a. Gegenstand einer Diskussion beim „Round Table Nutzung von Gesundheitsdaten zu Forschungszwecken unter der EU-Datenschutz-Grundverordnung (DSGVO)“, der am 03.07.2018 vom Bundesministerium für Wirtschaft und Energie mit über hundert Teilnehmenden - unter anderem aus den zuständigen Bundesministerien, vielen Landesministerien, der Wirtschaft (Pharma, Medizinprodukte- und Medizininformationsdienstleister), der medizinischen Versorgung und Forschung (u.a. TMF), Datenschutzbehörden und weiteren Institutionen aus der Fachöffentlichkeit - durchgeführt wurde. ${ }^{929}$

Die im Folgenden vorgestellten Regelungsvorschläge beruhen auf der Crundlage der bisher geführten Diskussion. Sie verfolgen das Ziel, den Vertraulichkeits- und Persönlichkeitsschutz von Patienten und Probanden in der medizinischen Forschung zu gewährleisten und gleichzeitig sicherzustellen, dass das wissenschaftliche Potenzial existierender Datenbestände so weit wie möglich ausgeschöpft wird. Dabei sind folgende grundsätzliche Erwägungen anzustellen:

Moderne Forschung ist immer mehr darauf angewiesen, räumlich und zeitlich auseinanderliegende Datenquellen mit unterschiedlicher Zweckbindung für eine gemeinsame Analyse zusammenzuführen. ${ }^{930}$

- Zur Sicherung der guten wissenschaftlichen Praxis müssen Forschungsergebnisse unabhängig nachvollziehbar sein, was wiederum die Aufbewahrung der diesen Ergebnissen zugrunde liegenden Daten in möglichst unverändertem Zustand voraussetzt.

- Angesichts der dynamischen Entwicklung von Erzeugung, Erfassung und Auswertung medizinischer Forschungsdaten sind die faktischen Möglichkeiten einer Anonymisierung zunehmend begrenzt.

Es bestehen heute technische Möglichkeiten (asymmetrische Kryptografie, homomorphe Verschlüsselung), die Verarbeitung von Forschungsdaten auf bestimmte Stellen und Zwecke zu begrenzen und so deren Vertraulichkeit zu wahren. ${ }^{931}$

Die Rechtszersplitterung in Deutschland sollte zugunsten eines möglichst einheitlichen Regelungsregimes beendet werden. ${ }^{932}$ Bei dieser länderübergreifenden Harmonisierung kann eine gestaffelte Melde- und Genehmigungspflicht für die Verarbeitung personenbezogener Forschungsdaten vorgesehen werden. Dadurch sollen gesetzliche Rahmenbedingungen geschaffen werden, mit denen die Einwilligung der Betroffenen als erforderliche Rechtsgrundlage ersetzt wird. ${ }^{933}$

Allerdings folgt die Gesetzgebungsbefugnis im Bereich der Forschung den jeweils zu regelnden Rechtsbereichen und den Zuständigkeiten für die tätigen Einrichtungen. Sie liegt daher sowohl beim Bund als auch bei den Ländern (s.o. Kap. 2.4). Wegen der

928 Krawczak/Weichert DANA 2017, $194 f$.

929 Weichert/Krawczak MIBE 2019, Vol. 15(1), 2/8.

930 Rfll, 4.

931 Weichert/Krawczak MIBE 2019, Vol. 15(1), 5/8; Krawczak/Weichert DANA 2017, 198.

932 Rfll, 10; GMDS, 3.

933 Datenethikkommission, 139 (These 17). 
im Grundgesetz festgeschriebenen geteilten Gesetzgebungskompetenzen kann eine einheitliche Regulierung durch einen Bund-Länder-Staatsvertrag erfolgen .934

Eine bundesweit einheitlich geltende Regelung für den Bereich der medizinischen Forschung ließe sich in der Praxis leichter durch eine Grundgesetzänderung erreichen. Darin sollte dann festgelegt werden, dass der Bereich des Datenschutzes in der medizinischen Forschung oder gar der Forschung generell in die (konkurrierende) Gesetzgebungskompetenz des Bundes übertragen wird. ${ }^{935}$ Zwar kann man die Ansicht vertreten, dass eine solche Gesetzgebungskompetenz schon durch die bestehenden Regelungen abgedeckt sei. Insbesondere Art. 74 Abs. 1 Nr. 13 GG, der die „Förderung der wissenschaftlichen Forschung“ dem Bundesgesetzgeber zuspricht, ließe sich nutzbar machen. ${ }^{936}$ Angesichts des Umstandes, dass es derzeit aber eine Vielzahl von Landesgesetzen gibt, die medizinische Forschung regeln, ist es zweifelhaft, dass die Bundesländer eine solche Zentralisierung der Normsetzungszuständigkeit ohne eine explizite Änderung des GG akzeptieren werden.

Erstrebenswert wäre darüber hinausgehend ein bundesweit geltendes Gesundheitsdatenschutzgesetz. ${ }^{937}$ Angesichts der Komplexität einer solchen Materie ist - zunächst - die Verwirklichung eines Medizinforschungsgesetzes realistischer. Angesichts der hohen Hürden für die Realisierung eines Bund-Länder-Staatsvertrags wird auch vorgeschlagen, ein Medizinisches Forschungsdatengesetz oder ein Gesundheitsdatennutzungsgesetz auf Bundesebene zu erlassen, dem sich die Bundesländer über Verweisungen anschließen können. ${ }^{938}$

Ein möglicher Ansatz für eine Harmonisierung besteht darin, dass auf Bundesebene keine umfassenden Regelungen erlassen werden, sondern lediglich regulatorische Festlegungen, etwa zur Federführung bei der Datenschutzaufsicht. ${ }^{939}$ Hierdurch werden jedoch nur einige Symptome der Rechtszersplitterung angegangen und keine praktische Vereinheitlichung erreicht.

Einen Weg zu einer untergesetzlichen Harmonisierung des Datenschutzes bei der medizinischen Forschung eröffnen die Art. 40, 41 DSGVO. Danach können Verbände oder andere Vereinigungen, die Kategorien von Verantwortlichen oder Auftragsverarbeitern vertreten, Verhaltensregeln ausarbeiten, mit denen eine Präzisierung der Regeln zur Verarbeitung personenbezogener Daten sowie des Umgangs damit festgelegt wird (Art. 40 Abs. 2 DSGVO). ${ }^{940}$ Der Vorteil solcher Regeln liegt darin, dass es für ihre Ausarbeitung keines aufwändigen Gesetzgebungsverfahrens bedarf. Deren Inhalt wird zwischen den beteiligten Stellen und den Datenschutzbehörden - auf der Grundlage der bestehenden Gesetze - ausgehandelt. Über solche Regeln können auch Stellen in Drittländern ohne angemessenes Datenschutzniveau einbezogen werden

934 Weichert/Krawczak MIBE 2019, Vol. 15(1), 6/8; Krawczak/Weichert DANA 2017, 199; ebenso Weichert in Steckler, Einzelaspekte rechtswissenschaftlicher Begleitforschung für Projekte der Mensch-Technik-Interaktion, 2019, 218ff.; kritisch bzgl. der praktischen Realisierbarkeit Dierks 2019, 89; Schneider, 344, schlägt eine Mustergesetzgebung vor, dazu Dierks 2019, $89 f$.

935 Kingreen/Kühling in Kingreen/Kühling, 468ff.; Dierks 2019, 103f.; vgl. Graf von Kielmansegg in TMF, $122 f$.

936 Dierks 2019, 90-100.

937 Dierks 2019, 112; Kingreen/Kühling in Kingreen/Kühling, 468ff.

938 Netzwerk Datenschutzexpertise; Sachverständigenrat, S. XXVIII; ähnlich Graf von Kielmansegg in TMF, 121.

939 Dierks 2019, 100-103; dem folgend, aber unsystematisch im SGB eingeordnet jetzt $\$ 287 a$ SGB V; dazu Sachverständigenrat 203; Schäfer in Kipker/Voskamp, $350 \mathrm{ff.}$

940 Dierks 2019, 104ff. 
(Art. 40 Abs. 3 DSGVO, s.o. Kap. 13.2). Die Reichweite solcher Verhaltensregeln ist skalierbar. ${ }^{941}$ Gesetzliche Vorgaben können mit Verhaltensregeln nicht abbedungen werden. Wohl aber können darüber Verfahren für den Umgang mit Normenkollisionen festgelegt werden. Vorlageberechtigt sind Vereinigungen von Daten verarbeitenden Stellen. ${ }^{942}$ Insofern kommt auch die TMF als „Normgeber“ in Betracht. Verhaltensregeln können für die erfassten Stellen verbindliche Vorgaben machen, die gemäß Art. 41 DSGVO überwacht werden. Sie bedürfen für ihre Verbindlichkeit der Genehmigung durch die zuständige Aufsichtsbehörde (Art. 40 Abs. 5 DSGVO). Verhaltensregeln könnten letztlich als Vorlage für spätere verbindliche gesetzliche Festlegungen dienen.

Bei der Schaffung einer einheitlichen materiellen Regulierung der Datennutzung für die medizinische Forschung kann auf die Grundsätze bestehender Forschungsklauseln zurückgegriffen werden, die sich in der Vergangenheit weitgehend bewährt haben.

- Soweit möglich, sind Daten für Forschungszwecke zu anonymisieren; ansonsten ist eine Pseudonymisierung vorzunehmen. Besteht später Bedarf an einer identifizierenden Zuordnung pseudonymer Datensätze, so kann über eine FileTrennung und eine unabhängige Vertrauensstelle gewährleistet werden, dass keine ungewollten Identifizierungen pseudonymisierter Datensätze erfolgen.

- Eine Verarbeitung personenbeziehbarer Forschungsdaten ist nur zulässig, wenn alle einschlägigen Schutzziele (Verfügbarkeit, Integrität, Vertraulichkeit, Transparenz, Intervenierbarkeit, Nichtverkettbarkeit) in angemessener Weise durch technisch-organisatorische Maßnahmen gewährleistet werden.

- Eine Verarbeitung ist zulässig, wenn sie auf einer ausdrücklichen, informierten, freiwilligen und widerrufbaren Einwilligung basiert. Die in der DSGVO enthaltenen Vorgaben ermöglichen Präzisierungen für die medizinische Forschung.

- Eine Verarbeitung kann auch ohne Einwilligung der Betroffenen zulässig sein, wenn ein öffentliches Interesse am jeweiligen Forschungsvorhaben besteht und der Schutz der Betroffenen gewährleistet werden kann.

- Personenbezogene Daten dürfen nicht veröffentlicht werden, es sei denn, die betroffene Person hat eingewilligt oder dies ist für die Darstellung der Forschungsergebnisse unerlässlich.

Die datenschutzrechtlichen Betroffenenrechte müssen stets so weit wie möglich gewährleistet werden. ${ }^{943}$

Forschung mit Berufsgeheimnissen, also insbesondere mit Patientengeheimnissen, erfordert wegen des Zwei-Schranken-Prinzips in Deutschland neben der Beachtung allgemeiner Datenschutzregelungen auch die Einhaltung der rechtlichen Anforderungen an die Verarbeitung von Berufsgeheimnissen. Diese Rechtslage sollte dahingehend geändert werden, dass an der Forschung Beteiligte unter bestimmten Bedingungen in einen an $\mathbb{} 203$ StCB orientierten Geheimnisschutz einbezogen werden. Ein gesetzliches Forschungsgeheimnis sollte ein Zeugnisverweigerungsrecht und

941 Weichert in DWWS, Art. 40 Rn. 17.

942 Dierks 2019, 109.

943 Weichert/Krawczak MIBE 2019, Vol. 15(1), 5/8. 
Beschlagnahmeverbot mit einschließen. ${ }^{944}$ Bei der Festlegung der Bedingungen sind Genehmigungen oder Zertifikate denkbar. ${ }^{945}$

Neben technisch-organisatorischen und rechtlichen Regelungen gibt es in den bestehenden Forschungsklauseln prozedurale Vorkehrungen wie z.B. Genehmigungsvorbehalte und Meldepflichten. Diese haben sich in der Praxis oft nicht bewährt. Der Prüfaufwand der beteiligten Stellen (Ministerien, Datenschutzaufsichtsbehörden, Ethikkommissionen) kann mit den vorhandenen Ressourcen nicht oder nur eingeschränkt erbracht werden.

Im Interesse der Entbürokratisierung und Vereinfachung wird demgegenüber ein Verfahren angeregt, in das technisch-organisatorische, datenschutzrechtliche, ethische und fachliche Erwägungen einfließen können, indem die erforderliche Expertise in unabhängigen, lokal agierenden Gremien (englisch: Use and Access Committees, UAC) im Sinne eines „One-Stop-Shopping“ gebündelt wird. Damit wird dem Bedürfnis Rechnung getragen, dass die Überprüfung der rechtlichen Zulässigkeit auf eine institutionelle Instanz übertragen wird, die sowohl die Sichtweise der Wissenschaft wie auch die der Beforschten bzw. des Datenschutzes berücksichtigt. ${ }^{946}$ Diesen UACs werden in Abhängigkeit von der Sensitivität des jeweiligen Forschungsvorhabens Genehmigungs- bzw. Vetorechte für die Nutzung personenbezogener Gesundheitsdaten per Gesetz übertragen, sie erhalten also eine hoheitliche Funktion. In den UACs muss fachlicher, ethischer und datenschutzrechtlicher Sachverstand vertreten sein.

Das Verhältnis der UACs zu den Ethikkommissionen und Datenschutzaufsichtsbehörden sollte unter Einräumung eines gegenseitigen Konsultationsrechts so geregelt werden, dass eine Kollision ihrer datenschutz- und berufsrechtlichen Compliance-, Kontroll- und Beratungspflichten weitestgehend vermieden und eine Entlastung der Beteiligten erreicht wird. Die Zuständigkeit eines UAC für ein bestimmtes Forschungsprojekt könnte sich aus der geographischen oder organisatorischen Zugehörigkeit des jeweils Projektverantwortlichen ergeben. Vorbild hierfür könnte die in der DSGVO verankerte Regelung zur Zuständigkeit der Aufsichtsbehörden sein, die sich an der Hauptniederlassung eines Verantwortlichen orientiert (Art. 56 Abs. 1 DSGVO). ${ }^{947}$

Während die organisatorischen und administrativen Verfahren der UACs gesetzlich zu regeln sind, sollten die Kriterien für die Bewertung von Forschungsvorhaben im Rahmen einer „regulierten Selbstregulierung“ durch Einrichtungen wie z.B. die TMF entwickelt werden. Die resultierenden Standards könnten im Konsens der betroffenen Fach-Communities auch als verbindlicher und rechtssicherer Rahmen für die Konzipierung und Zulassung von Forschungsvorhaben dienen. ${ }^{948}$ Bei diesen Standards können Treuhänderlösungen einen wesentlichen Beitrag leisten (s.o. Kap. 10.4).

944 GMDS, 7; Tinnefeld/Buchner/Petri/Hof, Einführung in das Datenschutzrecht, 7. Aufl. 2020, Rn. 523-525.

945 Krawczak/Weichert DANA 2017, 198.

946 Sachverständigenrat, 234, 327 (Rn. 761); RfIl, 13; Graf von Kielmansegg in TMF, 121f.; tendenziell ebenso Deutscher Ethikrat, 183 (D2.1); Platzer NSZ 2020, 94; Dierks 2020, 11ff.; zur ethischen Bewertung Strech in TMF, $72 f f$.

947 Krawczak/Weichert, DANA 2017, 199.

948 RfIl, 14; generell dazu Datenethikkommission, 29 (These 58), 201ff.; Weichert 2018, Kap. 8.22; Health Ethics Policy Lab, $72 f f$. 
Ein wichtiger Baustein kann auch in der Zertifizierung von Einzellösungen, in standardisierten Prozessen und in der Erarbeitung von Verhaltensregeln (Codes of Conduct) liegen (vgl. Art. 42, 43 DSGVO). ${ }^{949}$

Derzeit gibt es im Kontext der Verarbeitung von personenbezogenen Daten für medizinische Forschungszwecke keine demokratische Kontrolle; den entsprechenden Verfahren fehlt systematische Transparenz (s.o. Kap. 3.4). Bei einer (teilweisen) Bündelung der bisherigen Aufgaben von Ministerien, Aufsichtsbehörden und Ethikkommissionen in eigens dafür eingerichteten und untereinander vernetzten UACs ließe sich dieser Missstand durch den Betrieb eines öffentlich einsehbaren Forschungsregisters beheben, an das die UAC wesentliche Informationen zu den von ihnen freigegebenen Forschungsprojekten weitergeben. Dieses Register wäre im Interesse eines wissenschaftlichen Dialogs eine Informationsgrundlage für andere Forschende. Es könnte zudem den von den Forschungsprojekten Betroffenen einen Überblick über die Forschung mit ihren Daten, die dafür jeweils Verantwortlichen, ihre Ziele und Fragestellungen sowie die in der Forschung ergriffenen grundrechtsschützenden Maßnahmen erlauben. Nicht zuletzt könnte damit auch der immer wieder erhobenen Forderung nach stärkerer Teilhabe der Patienten und Probanden und der Möglichkeit für diese, Widerspruch einzulegen, Rechnung getragen werden.950

Die den Forschenden abzuverlangende Transparenz sollte gesetzlich möglichst präzise so festgelegt werden, dass einerseits die wissenschaftliche Nutzung der personenbezogenen Daten hinreichend nachvollziehbar und überprüfbar ist, dass andererseits den Forschenden der größtmögliche wissenschaftliche Freiraum bewahrt wird. Bezüglich des Forschungszweckes erscheint eine Offenlegung der Fragestellung, der eingesetzten Methoden und Verfahren und der damit verbundenen Datenverarbeitung (Forschungs- bzw. Datenschutzkonzept) gegenüber einem gesetzlich legitimierten Cremium, etwa dem UAC, ausreichend, das die Befugnis zur Bewertung und zur Genehmigung des Projektes hat.

Wird ein Forschungsprojekt nicht auf Einwilligungsbasis durchgeführt, so sollte mit der Antragstellung im Interesse der Transparenz gegenüber Betroffenen in allgemeiner Form eine Veröffentlichung, z.B. über ein Internetportal, zur Pflicht gemacht werden. ${ }^{951}$ Wurde ein entsprechendes Vorhaben zugelassen und begonnen, so ist dies öffentlich zu vermerken. Dabei sollte auch erkennbar gemacht werden, für wann der Abschluss des Projektes geplant ist; bei langfristigen Forschungsprojekten können auch Angaben zu beabsichtigten Zwischenzielen sinnvoll sein. Nach Abschluss eines Projektes sollte nach einer bestimmten Frist eine allgemeine Veröffentlichung der Ergebnisse zur Pflicht gemacht werden. Insofern erscheint eine Frist von einem Jahr nach Projektabschluss angemessen.

Durch eine bundesweit einheitliche Regelung könnten die bisherigen, teilweise verstreuten und widersprüchlichen Bundes- und Länderregelungen zur medizinischen Forschung ersatzlos wegfallen. Darin müssten die materiell-rechtlichen und prozeduralen Voraussetzungen für die Zulässigkeit medizinischer Forschungsvorhaben normiert werden - einschließlich eventueller Einwilligungserfordernisse, der

949 EDPS 2020, 25f.; RfII, 15, 19, 23.

950 Datenethikkommission, 126; Krawczak/Semler/Zenke/Strech/Graf von Kielmansegg in TMF, 132.

951 Ähnlich für Forschungsprojekte auf der Basis des Forschungsdatenzentrums $\$$ 303d Abs. 1 Nr. 6 SGB V. 
Verfahren der UACs, der Einbindung von Ethikkommissionen und Datenschutzaufsicht sowie der Transparenzverpflichtungen gegenüber der Öffentlichkeit.

Die Anregung zur Einrichtung unabhängiger UACs für medizinische Forschungsdaten basiert neben inhaltlichen Erwägungen auch auf der Notwendigkeit, Doppelentwicklungen und Parallelstrukturen in diesem wichtigen und sensiblen Bereich zu vermeiden. Seit Juli 2017 fördert das Bundesministerium für Bildung und Forschung (BMBF) umfänglich die Medizininformatik-Initiative, in der deutsche Universitätskliniken gemeinsam mit externen Partnern sogenannte „Datenintegrationszentren“ (DIZ) zum standortübergreifenden Managen und Teilen medizinischer Daten aufbauen. Die geförderten Konsortien sehen in ihren Konzepten in der einen oder anderen Weise Mechanismen vor, um den Zugang zu den zu teilenden Daten formal auszugestalten. ${ }^{952}$ Die Etablierung solcher Verfahren wird voraussichtlich zentral für die Funktionsfähigkeit der DIZ sein. Aus der Medizininformatik-Initiative können umfassendere Verfahren entwickelt und etabliert werden. ${ }^{953}$

Welche Forschungsprojekte unabhängig vom Datenzugang melde- bzw. genehmigungspflichtig sein sollen bzw. können, bedarf der weiteren fachlichen Erörterung. Maßgebliches Kriterium soll dabei das mit dem jeweiligen Projekt verbundene Datenschutzrisiko sein.

- Auf eine Meldung und Registrierung kann verzichtet werden, wenn klassische Eigenforschung erfolgt oder die Forschungsdatenverarbeitung auf einer informierten Einwilligung der Betroffenen basiert.

- Melde- und registrierungspflichtig sollten Projekte sein, bei denen eine Interessenabwägung die informierte Betroffeneneinwilligung ganz oder teilweise ersetzen soll, was impliziert, dass die UACs bei solchen Projekten neben Aufklärungs- auch Untersagungsrechte haben müssen.

- Zusätzlich zur bestehenden Meldepflicht sollten Projekte genehmigungspflichtig sein, wenn in ihnen hochsensitive Daten verarbeitet werden, wie dies z.B. bei umfangreichen Gensequenzierungen der Fall ist, oder wenn weiterreichende Zweckänderungen beabsichtigt sind. Auch zeitlich unbegrenzte Studien bzw. Forschungsdatenbanken sollten unter Genehmigungsvorbehalt gestellt werden.

Für ethisch oder technisch besonders anspruchsvolle Projekte wie z.B. internationale Studien, Forschungsnetzwerke, Krankheitsregister oder Biomaterialdatenbanken könnten vom zuständigen UAC bei Bedarf zusätzliche Anforderungen festgelegt und zur Genehmigungsgrundlage gemacht werden. ${ }^{954}$

Das vorgeschlagene Regelungsverfahren trägt zu einer Optimierung des Datenschutzes bei medizinischen Forschungsprojekten bei und erleichtert und verbessert zugleich die Forschungspraxis. Die Umsetzung des Konzepts ist nicht vom Schreibtisch aus möglich. Vielmehr ist hierfür eine umfassende Fortführung des begonnenen Diskussions- und Abstimmungsprozesses unter Einbindung aller Betroffenen erforderlich. Die genaue Ausgestaltung der UACs muss auf den in der Vergangenheit

952 Der Bayerische Landesbeauftragte für den Datenschutz, 28. TB, 2017/2018, 108ff.; Zenker/Krawczak/Sempler in TMF, 43ff.

953 Netzwerk Datenschutzexpertise, 7f.; generell zur Initiative Sachverständigenrat, 37f., 211.

954 Weichert/Krawczak MIBE 2019, Vol. 15(1), 7 u. 8; Weichert ZD 2020, $23 f$. 
gemachten Erfahrungen basieren und einem strukturierten Prozess folgen, an dessen Ende eine gesetzliche Festlegung stehen sollte. Wie vom Rat für InformationsInfrastrukturen (RfII) und jüngst vom Sachverständigenrat gefordert, sollte dieser Entwicklungsprozess Hand in Hand mit dem Aufbau einer netzwerkförmigen Nationalen Forschungsdateninfrastruktur (NFDI) erfolgen. 955

\subsection{Europäische Reformmöglichkeiten}

Angesichts der zunehmenden Europäisierung der Forschung generell wie der medizinischen Forschung speziell und dem damit verbundenen Austausch personenbeziehbarer Informationen innerhalb der EU und mit Projekten außerhalb der EU wäre nicht nur eine einheitliche nationale Regulierung der Forschungsdatenverarbeitung wünschenswert, sondern darüber hinausgehend eine verbindliche europäische Regelung. ${ }^{956}$

Zwar besitzt die EU keine Regelungskompetenz im Hinblick auf Forschungsfragen generell. Hinsichtlich der Normierung der personenbezogenen Datenverarbeitung für Forschungsprojekte sowie der Verarbeitung in solchen Projekten hat die Union über Art. 16 Abs. 2 AEUV aber eine umfassende Zuständigkeit (s.o. Kap. 2.3). Gegen eine unionsweite Regulierung kann der Subsidiaritätsgrundsatz (Art. 5 EUV) schwerlich in Stellung gebracht werden. Eine nationale bzw. regionale Differenzierungsmöglichkeit besteht im Hinblick auf die Anforderungen an einen europäischen „Forschungsbinnenmarkt" nicht.

Dies gilt insbesondere für die medizinische Forschung, zumal die zu beantwortenden Forschungsfragen von nationalen Grenzen weitestgehend unabhängig sind. Möglich wäre eine umfassende europäische Regulierung des Datenschutzes im Rahmen der wissenschaftlichen Forschung. Möglich wäre aber auch eine Beschränkung auf die medizinische Forschung: Zwar bestehen zwischen der medizinischen Forschung und anderen Forschungsbereichen, etwa im Bereich der Umwelt- und der Sozialwissenschaft, Wechselbeziehungen und disziplinübergreifende Ansätze. Doch ist die personenbezogene Datenverarbeitung in der medizinischen Forschung, einschließlich der wissenschaftlichen Behandlung der Biotechnik, ein Sektor, der weitgehend separat reguliert werden kann und derzeit im Hinblick auf spezifische Anwendungen auch auf nationaler Ebene quellenbezogen separat geregelt ist.

Als Grundlage für eine europäische Harmonisierung der Regelungen zur (medizinischen) Forschung mit personenbezogenen Daten genügt die DSGVO mit ihrer Privilegierung von Forschungszwecken und dem Erfordernis kompensierender Garantien für die Betroffenen. Die einschlägigen Öffnungsklauseln, etwa in Art. 6 Abs. 3 u . 4, 9 Abs. 2 lit. j, 23, 89 Abs. 2 u. 3 DSGVO, eröffnen nicht nur den Mitgliedstaaten, sondern auch der Union selbst die Befugnis zur Konkretisierung der allgemeinen Vorgaben in der DSGVO. Eine Harmonisierung auf Unionsebene hätte zudem den Effekt, dass verbindliche Regelungen für Deutschland auf Bundes- wie auf Landesebene

955 RfIl, 15ff.; Deutscher Ethikrat, 174 (A1); Sachverständigenrat, 231ff.; generell zum Aufbau von Dateninfrastrukturen Datenethikkommission, 143.

956 Datenethikkommission, 32, 125, 139 (These 17), 226ff.; Platzer NZS 2020, 295; Weichert ZD 2020, 18; Martini/ Hohmann NJW 2020, 3578; ausführlich Dierks 2020, $5 \mathrm{ff}$. 
geschaffen würden, da das EU-Recht dem Recht auf beiden Ebenen vorgeht. Dies hätte den Vorteil, dass das derzeit auf nationaler Ebene bestehende Problem unterschiedlicher Gesetzgebungskompetenzen (s.o. Kap. 2.4, Kap. 14.1) keine Rolle spielen würde. Die ungenügende Umsetzung der Vorgaben der DSGVO (s.o. Kap. 14.1) im Bereich der Forschung würde überregelt. Die unübersichtliche Annexregulierung der Forschung in vielen unterschiedlichen Normtexten ließe sich überwinden (s.o. Kap. 2.4). Die Zweigleisigkeit der nationalen Regelung mit dem Datenschutzrecht und dem Berufsgeheimnisrecht (s.o. Kap. 6, Kap. 14.1) könnte zusammengeführt werden.

Als Regelungsinstrument bietet sich eine Verordnung an. Eine Richtlinie, die wieder in nationales Recht umzusetzen wäre, hätte nicht die wünschenswerte Harmonisierungswirkung. Als Übergangsregelung wäre ein Code of Conduct (Verhaltensregeln) gemäß Art. 40 DSGVO denkbar. ${ }^{957}$ Das Problem solcher Verhaltensregeln bestünde darin, dass sie sich zwangsläufig in Widerspruch setzen müssten zu bestehenden nationalen gesetzlichen Regelungen, die in der Normhierarchie über Verhaltensregeln anzusiedeln sind. ${ }^{958}$ Rechtfertigen ließe sich ein solches Vorgehen allenfalls damit, dass diese Verhaltensregeln Vorgaben zu einer DSGVO-konformen Umsetzung des bestehenden Rechts machen. Für nationale Sonderwege sind keine Notwendigkeiten erkennbar. Sollten sich diese in der weiteren Diskussion ergeben, kann man, dem Vorbild der DSGVO folgend, über Öffnungsklauseln eng beschränkte Regelungsspielräume schaffen.

Bezüglich des Regelungsinhaltes kann weitgehend auf die Ausführungen unter Kapitel 14.2 verwiesen werden. Die gemeinsamen Grundannahmen bestehen für die gesamte Union mit der Grundrechte-Charta; diese Grundrechte entsprechen denen des deutschen Verfassungsrechts. Zudem bestehen internationale Standards, an die angeknüpft werden kann (s.o. Kap. 7.2). Soweit erkennbar, bestehen hinsichtlich der Grundannahmen zwischen den Rechtsordnungen innerhalb der EU keine wesentlichen Unterschiede. Inwieweit nationale Besonderheiten bestehen, muss eventuell durch Studien im Detail abgeklärt werden. Der Umstand, dass sich über die Forschungsförderung der EU in der Gemeinschaft der Forschenden schon gemeinsame Standards entwickelt haben, ist für die Konkretisierung der Forschungsdatenverarbeitung förderlich.

Politische Initiativen zur Harmonisierung des Datenschutzrechts bei der Forschung sind bisher nicht erkennbar. Es ist aber auch nicht ersichtlich, dass nationale oder interessenspezifische Interessen einer solchen Harmonisierung im Wege stehen. Die Chancen für die Entwicklung eines einheitlichen Forschungsraumes in der EU für Wissenschaft, Politik und Wirtschaft dürften allen Beteiligten einleuchten.

957 s. hierzu den umfassenden Vorschlag von Dierks 2020, 31ff., mit dem Titel „Europäischer Schutzraum für Forschungsdaten".

958 Dierks 2020, 35. 


\subsection{Fazit: Novellierungsbedarf}

Der Wissenschaftsstandort Deutschland leidet im internationalen Wettbewerb seit Jahren unter dem Fehlen einheitlicher gesetzlicher Rahmenbedingungen, die ein zukunftsgerichtetes Forschen mit Gesundheitsdaten unter gleichzeitiger Wahrung der Grundrechte der betroffenen Menschen ermöglichen. Dadurch ergeben sich Nachteile für die wirtschaftliche Entwicklung, den gesellschaftlichen Fortschritt und den Grundrechtsschutz der Menschen. Durch die Vorschläge zur Regulierung und durch den parallelen Aufbau einer entsprechenden Infrastruktur könnte diese Blockade aufgelöst werden. Im föderalen Deutschland gesammelte Erfahrungen können in dem größeren Rechtsraum der EU sowie darüber hinausgehend international nutzbar gemacht werden.

Ohne eine Vermittlung dieser Anliegen gegenüber der Öffentlichkeit wird es nicht möglich sein, die nötige Einsicht in die Notwendigkeit einer Weiterentwicklung des rechtlichen Rahmens für die medizinische Forschung bei den politisch Verantwortlichen zu schaffen und die nötige Akzeptanz dafür zu gewinnen, dass die sensitiven Daten der Bevölkerung im Interesse des Gemeinwohls beforscht werden. ${ }^{959}$ Datenschutz und Forschung sind beides Gemeinwohlanliegen. Grundlage für das Einwirken auf die öffentliche Meinung sollte es sein, dass sich die Gemeinschaft der Forschenden und die der Datenschützer auf gemeinsame Positionen verständigen. Hierzu muss der Austausch zwischen diesen Beteiligten intensiviert werden. ${ }^{960}$ 